\title{
PHENOMENOLOGY OF SCALAR MESONS
}

\author{
Leonard Leśniak \\ H. Niewodniczański Institute of Nuclear Physics, \\ PL 31-342 Kraków, Poland
}

\begin{abstract}
A separable potential model of three coupled channels $\pi \pi, K \bar{K}$ and an effective $2 \pi 2 \pi$ has been constructed and applied in the analysis of the isoscalar S-wave $\pi \pi$ and $K \bar{K}$ phase shifts and inelasticities. A relatively narrow scalar resonance of mass 1400-1460 MeV has been found. This resonance is consistent with the recent CERN observation of an eventual scalar glueball at $1500 \mathrm{MeV}$. Other resonances at lower energies like a wide $\sigma$ meson and a narrow $f_{0}(980)$ have been confirmed.
\end{abstract}

Scalar meson spectroscopy is full of open questions. The internal structure of scalars is also very controversial, for example the $f_{0}(980)$ state is interpreted as $q \bar{q}, q \bar{q} q \bar{q}$ or $K \bar{K}$ quasibound state. Mixing of scalar mesons with scalar glueballs is possible; for example the $f_{0}(1500)$ resonance, observed by the Crystal Barrel Collaboration in $\bar{p} p$ annihilation at CERN, was interpreted as a candidate for the lowest scalar glueball. The phenomenology of scalar mesons must include studies of interactions between different mesons produced in the decay channels of scalar mesons. In Refla the scalar meson spectrum was studied in terms of a relativistic $\pi \pi$ and $K \bar{K}$ coupled channel model from the $\pi \pi$ threshold up to $1400 \mathrm{MeV}$. The phenomenological parameters were constrained by fitting the $S$-wave data extracted from the experimental cross sections on the $\pi^{+} \pi^{-}$production by $\pi^{-}$scattering on unpolarized hydrogen target ${ }^{3}$ and further constraints were imposed by the $K \bar{K}$ phase shift analysis of Ref.

Recently, authors of Ref. ${ }^{3}$ have analysed data obtained on a polarized target by the CERN-Cracow-Munich group for the $\pi^{-} p_{\uparrow} \rightarrow \pi^{+} \pi^{-} n$ reaction. Separation of the $\pi$ and $a_{1}$ exchange amplitudes in this reaction was then possible for the first time. From a set of four solutions for the isoscalar $S$-wave phase shifts up to $1600 \mathrm{MeV}$, two of them ("down-flat" and "up-flat") satisfy the unitarity constraint. The "down-flat" solution is in good agreement with the former solution of Ref 3 up to $1400 \mathrm{MeV}$. Above 1400 $\mathrm{MeV}$ one observes an increase of the phase shifts and larger inelasticities than those seen in 3 . This could be a manifestation of the presence of scalar mesons $f_{0}(1370)$ or $f_{0}(1500)$ in that energy range. There a strong four-pion production has been observed in different experiments together with an evidence of clustering into $\sigma \sigma$ or $\rho \rho$ pairs.

In Ref.6 we have extended the isospin $0 S$-wave relativistic 2-channel model of Ref. 2 by adding to its $\pi \pi$ and $K \bar{K}$ channels an effective third coupled channel, 
here called $\sigma \sigma$. The reaction amplitudes $T$ satisfy a system of the coupled channel Lippmann-Schwinger equations:

$$
T=V+V G T
$$

where $V, G$ and $T$ are $3 \times 3$ matrices, $V$ is the interaction matrix and $G$ is the diagonal matrix of channel propagators:

$$
G_{j}\left(E, k_{j}\right)=\frac{1}{E-2 E_{j}\left(k_{j}\right)+i \epsilon}, \quad \epsilon \rightarrow 0(+), \quad j=1,2,3 .
$$

In Eq. (2) $E$ is the total energy, $E_{j}=\sqrt{m_{j}^{2}+{k_{j}}^{2}}$ and $m_{j}$ is the meson mass in channel $j$. We consider meson pairs of same mass and momentum $k_{j}$ in their centre of mass system. In this way 9 meson-meson reactions are simultaneously described by Eq.(1):

$$
\left(\begin{array}{ccc}
\pi \pi \rightarrow \pi \pi & \pi \pi \rightarrow K \bar{K} & \pi \pi \rightarrow \sigma \sigma \\
K \bar{K} \rightarrow \pi \pi & K \bar{K} \rightarrow K \bar{K} & K \bar{K} \rightarrow \sigma \sigma \\
\sigma \sigma \rightarrow \pi \pi & \sigma \sigma \rightarrow K \bar{K} & \sigma \sigma \rightarrow \sigma \sigma
\end{array}\right)
$$

We choose a separable form of the interaction:

$$
<p\left|V_{i j}\right| q>=\sum_{\alpha=1}^{n} \lambda_{i j, \alpha} g_{\alpha, i}(p) g_{\alpha, j}(q), \quad i, j=1,2,3
$$

where $\lambda_{i j, \alpha}$ are coupling constants and

$$
g_{\alpha, j}(p)=\sqrt{\frac{4 \pi}{m_{j}}} \frac{1}{p^{2}+\left(\beta_{\alpha, j}\right)^{2}}
$$

are form factors which depend on the relative centre of mass meson momenta $p$ in the final channel or $q$ in the initial channel. In the $\pi \pi$ channel $(j=1)$ we choose a rank-2 separable potential $(n=2)$ and in the other channels a rank-1 potential $(n=1)$. Altogether, this model has 13 parameters: 9 coupling constants $\lambda_{i j, \alpha}$ and 4 range parameters $\beta_{\alpha, j}$.

The model is unitary. The diagonal S- matrix elements are parametrized as

$$
S_{j j}=\eta_{j} e^{2 i \delta_{j}}, \quad j=1,2,3,
$$

where $\eta_{j}$ and $\delta_{j}$ are channel $j$ inelasticities and phase shifts, respectively. Expressions for nondiagonal elements can be found in Ref. S. Some of the $S$-matrix poles in the complex energy plane can be interpreted as resonances. We fit the existing experimental results on the $\pi \pi S$-wave isoscalar phase shifts together with inelasticity in the $\pi \pi$ channel and with the $K \bar{K}$ phase shifts.

Below $600 \mathrm{MeV}$ we have used data from the $K_{e 4}$ decay and above $600 \mathrm{MeV}$ the "down-flat" and "up-flat" solutions of the analysis of Ref.5 on a polarized target. In addition we have used the results of the analysis of reactions $\pi^{-} p \rightarrow K^{+} K^{-} n$ and $\pi^{+} n \rightarrow K^{-} K^{+} p$, although targets were unpolarized there $\oplus$. Four fits for $\delta_{\pi \pi}$ and $\eta_{\pi \pi}$ 
are compared to the experiment in Fig. 1. Above $1400 \mathrm{MeV}$ both "down-flat" and "up-flat" data indicate a decrease towards small values of $\eta$ ( $\eta \approx 0.6$ to 0.7 ), albeit with large errors. One can notice a better agreement with data of the 3-channel model in comparison with the 2-channel one. The main difference between the 2- and 3channel fits lies in $\eta$ above $1400 \mathrm{MeV}$, where the opening of the $\sigma \sigma$ channel leads to a fast decrease of inelasticity parameters. In order to achieve this behaviour, couplings between the $\pi \pi$ and $\sigma \sigma$ or $K \bar{K}$ channels should be sizable. Fits of similarly good quality were obtained with very different physical parameters in the $K \bar{K}$ and $\sigma \sigma$ channels. Lack of a sufficient number of observables and/or experimental precision, in particular in the effective $4 \pi$ channel, leads to the existence of several good sets of model parameters with quite different channel and interchannel interactions.

We have studied positions of the $S$-matrix poles in the complex energy plane $(E=M-i \Gamma / 2)$. These positions have not been parameters of our fits. In our model we have not included any kind of arbitrary background to fit the data. We have only fitted the meson-meson interaction parameters and then analyzed the analytical structure of the reaction amplitudes. At low energy we find a very broad $f_{0}(500)$ resonance (also called $\sigma$ meson) of a width between 510 and $560 \mathrm{MeV}$. In the 3 -channel fits the $f_{0}(980)$ resonance is seen in a vicinity of the $K \bar{K}$ threshold with a width of about 60 to $70 \mathrm{MeV}$. The relatively narrow state $f_{0}(1400)$ appears in the 3 -channel fits. Its mass varies from about $1400 \mathrm{MeV}$ to $1460 \mathrm{MeV}$ and the width is about $150 \mathrm{MeV}$. This width is close to values found by the Crystal Barrel Group (Ref.1), however the resonance masses obtained in our fits are smaller than their values of about $1500 \mathrm{MeV}$.

Finally, let us mention an important difference between the fits presented here for data taken on a polarized target in comparison with those performed with data 3 obtained on a nonpolarized target. If we fit the data of Ref 3 using our 3-channel model, we obtain a very wide resonance at $M=1521 \mathrm{MeV}$ of width $503 \mathrm{MeV}$. This means that the recent analysis of data 5 supplies new information on the $f_{0}(1400)$ meson.

In conclusion, we have analysed the isoscalar S-wave $\pi \pi$ and $K \bar{K}$ scattering using the CERN-Cracow-Munich data5 in the framework of the 2- and the 3-channel models of meson-meson scattering. All fits of the phase shifts analysis indicate presence of a relatively narrow scalar resonance of mass $1400-1460 \mathrm{MeV}$. This resonance is quite compatible with recent observations of a possible scalar glueball at $1500 \mathrm{MeV}$ with a width of $100 \mathrm{MeV}$.

This work has been performed in the framework of the IN2P3 - Polish Laboratories Convention (project No 93-71).

\section{References}

1. A. Abele et al., Phys. Lett. B385 (1996) 425.

2. R. Kamiński, L. Leśniak and J.-P. Maillet, Phys. Rev. D50, 3145 (1994).

3. G. Grayer et al., Nucl. Phys. B75, 189 (1974).

4. D. Cohen et al., Phys. Rev. D22 (1980) 2595.

5. R. Kamiński, L. Leśniak and K. Rybicki, Z. Phys. C74, 79 (1997).

6. R. Kamiński, L. Leśniak and B. Loiseau, Phys. Lett. B413, 130 (1997).

7. L. Leśniak, Acta Phys. Pol. B27, 1835 (1996). 

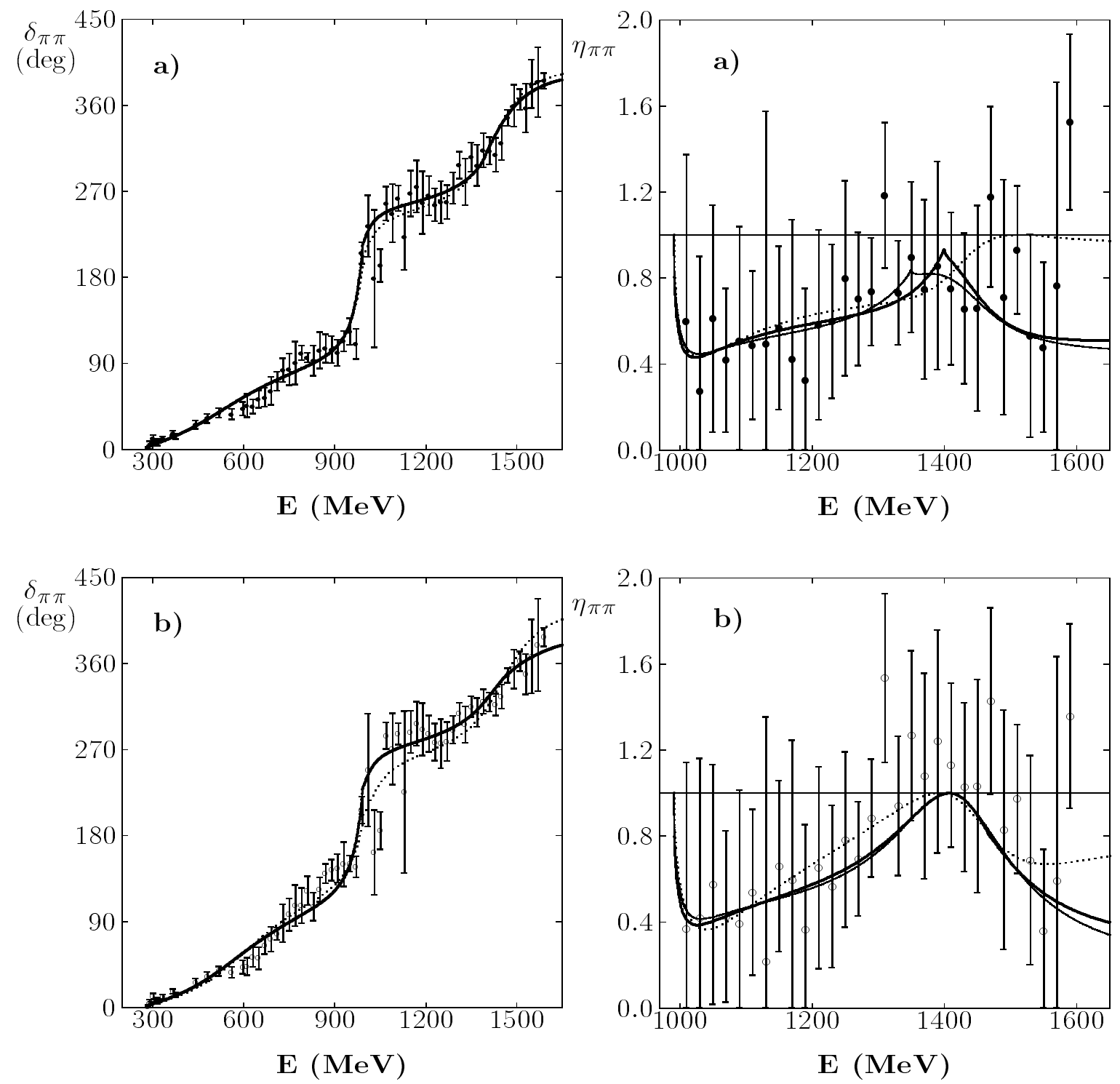

Fig. 1 Energy dependence of $\pi \pi$ phase shifts and inelasticities: a) fit to "down-flat" data of ref. 3 , thick solid line corresponds to fit A, thin solid line to fit B and dotted line to the 2-channel model fit; b) fit to "up-flat" data of ref5, thick solid line corresponds to fit C, thin solid line to fit D and dotted line to the 2-channel model fit. 